\title{
Effect of dabai (Canarium odontophyllum) fruit extract on biochemical parameters of induced obese-diabetic rats
}

\begin{abstract}
Dabai is one of the most popular indigenous fruits in Sarawak, and is rich in various nutrients. In this study, the effect of dabai fruit extract $(300$ and $600 \mathrm{mg} / \mathrm{kg} \mathrm{bw})$ on the biochemical parameters of obese-diabetic $(\mathrm{Ob}-\mathrm{db})$ rats was investigated. After a 4-week treatment period, the dabai extract at a concentration of $600 \mathrm{mg} / \mathrm{kg}$ bw (equivalent to $20 \mathrm{~g}$ of flesh with skin of fresh fruit) showed a pronounced effect in lowering the plasma glucose level compared with $300 \mathrm{mg}$ extract $/ \mathrm{kg}$ bw (equivalent to $10 \mathrm{~g}$ of flesh with skin of fresh fruit). The dabai extract also significantly reduced the plasma cholesterol and low-density lipoprotein (LDL-c) levels and increased high-density lipoprotein (HDL-c). However, the dabai extract did not increase the insulin level but did increase its sensitivity and reduced insulin resistance (HOMA-IR). This study suggests that dabai extract possesses hypocholesterolaemic properties, has a shortterm glucose-lowering effect and improves the lipid profile through synergistic effect of various polyphenols.
\end{abstract}

Keyword: Dabai; Obese-diabetic; Polyphenol compounds; Glucose; Insulin; Lipid profile 